\title{
Dynamic modeling and analysis of the large-scale rotary machine with multi-supporting
}

\author{
Xuejun Li, Yiping Shen* and Songlai Wang \\ Hunan University of Science and Technology, Hunan Provincial Key Laboratory of Health Maintenance for \\ Mechanical Equipment, Hunan, China
}

Received 8 February 2010

Revised 5 May 2010

\begin{abstract}
The large-scale rotary machine with multi-supporting, such as rotary kiln and rope laying machine, is the key equipment in the architectural, chemistry, and agriculture industries. The body, rollers, wheels, and bearings constitute a chain multibody system. Axis line deflection is a vital parameter to determine mechanics state of rotary machine, thus body axial vibration needs to be studied for dynamic monitoring and adjusting of rotary machine. By using the Riccati transfer matrix method, the body system of rotary machine is divided into many subsystems composed of three elements, namely, rigid disk, elastic shaft, and linear spring. Multiple wheel-bearing structures are simplified as springs. The transfer matrices of the body system and overall transfer equation are developed, as well as the response overall motion equation. Taken a rotary kiln as an instance, natural frequencies, modal shape, and response vibration with certain exciting axis line deflection are obtained by numerical computing. The body vibration modal curves illustrate the cause of dynamical errors in the common axis line measurement methods. The displacement response can be used for further measurement dynamical error analysis and compensation. The response overall motion equation could be applied to predict the body motion under abnormal mechanics condition, and provide theory guidance for machine failure diagnosis.
\end{abstract}

Keywords: Multi-supporting, axis line, body vibration, anisotropy stiffness, Riccati transfer matrix method

\section{Introduction}

The larger-scale rotary machines, such as rotary kiln, rope laying machine, cylinder drier, and cooling machine, are widely used for material calcination, stranding, drying, and cooling in the architectural, chemistry, and agriculture industries. Their main bodies of these machines are supported by many groups of wheel- bearing supporting structures, as shown in Fig. 1. These machines are generally large scale, over-weight, and statically indeterminate. Axis line deflection is a vital parameter to determine mechanics state of rotary machine, that is well investigated in Refs. [1-4]. Little axis line deflection can cause large difference load distributed between each wheel. It is reported that when the axis line deflection is $\pm 10 \mathrm{~mm}$, the body stress increases three times, and the load applied on the supporting wheel increases one time [2,5]. Body axial vibration are excited when asymmetric geometry occurs caused by abrasion and thermal expansion, machine runs abnormally, and driveline vibrates. This brings dynamic stress to all parts and causes severe hazard to the machine. Therefore, body axial vibration of the large-scale rotary machine with multi-supporting should be paid sufficient attention. In practice, as for a rotary kiln, axial line measurement is every-

\footnotetext{
${ }^{*}$ Corresponding author: Yiping Shen, E-mail: yiping1011@163.com.
} 

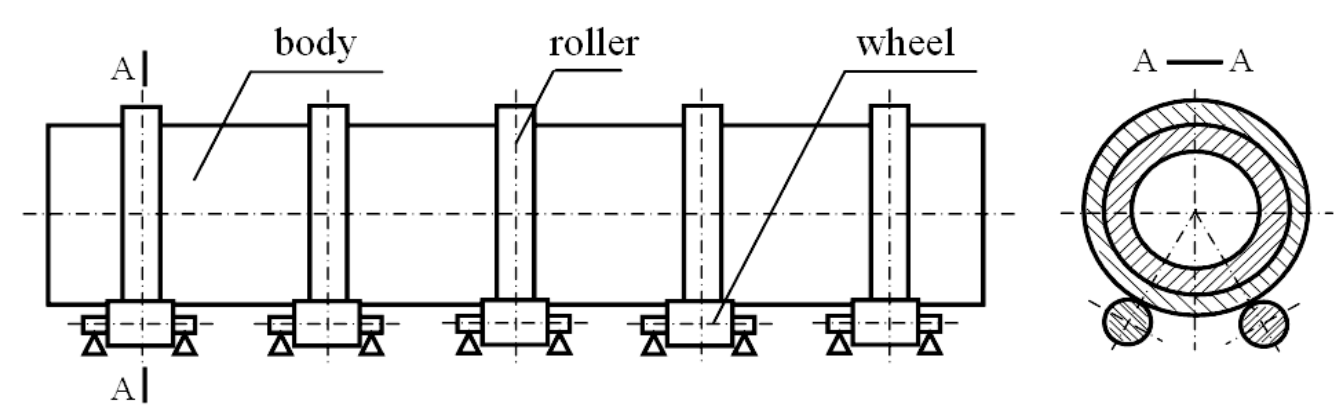

Fig. 1. The sketch of the large-scale rotary machine with multi-supporting.
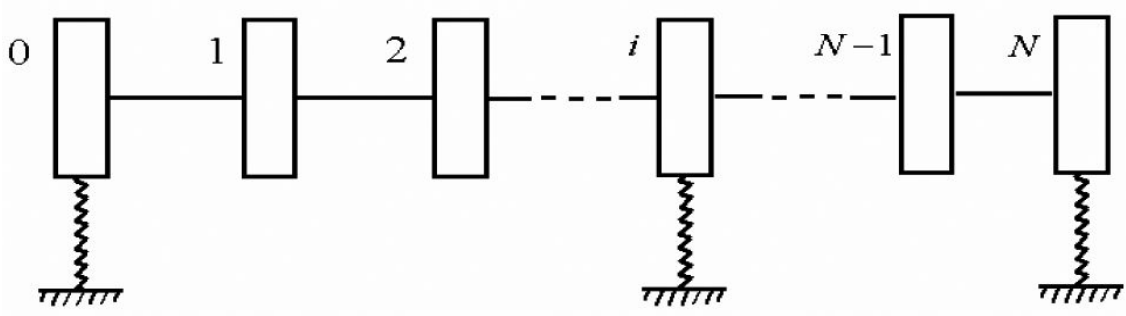

Fig. 2. Discrete model with $N$ subsystems.

day radical task to maintain the equipment [6-10]. However, dynamical measurement error is found to be important for efficient and accurate adjustment. Thus, it is necessary to perform dynamical modeling and analysis of this kind of rotary machine.

The body, rollers, wheels, and bearings of rotary machine constitute a chain multibody system. A lot of methods for chain multibody system dynamics have been studied by many researchers [11,12]. The transfer matrix method (TMM) has been developed for a long time and has been applied widely in structure mechanics and multibody system dynamics [13]. The advantage of TMM is that the global dynamics equations of the system are not needed and the matrix order does not increase with the number of elements. Holzer firstly used TMM to solve the problems of torsion vibration of rods [14]. Prohl applied TMM for rotor system dynamics [15]. Horner proposed Riccati transfer matrix method (Riccati TMM) to improve numerical stability [16]. Dokanish proposed the finite element-transfer matrix method to solve plate structure vibration problem [17]. Kumar and Sankar developed discrete time transfer matrix method(DTTMM) for time variant system dynamics [18]. In recently years, many researchers continuously developed TMM to a wide variety of engineering problems [19-27].

In this investigation, Riccati transfer matrix method is used to analyze the dynamics of the body system with multiple wheel-bearing supporting structures. This paper is organized as follows. In Section 2, the body is divided into many subsystems with the deduced stiffness coefficients of multiple wheel-bearing supporting structures, and transfer matrices and overall equation are established. In Section 3, the Riccati transform equation is introduced to improve numerical stability for computing natural frequencies and modal shape of the body system. The response overall transfer equation is given in Section 4. In Section 5, a rotary kiln as an example is presented with the numerical results, and then some conclusions are given.

\section{Discretization of the large-scale rotary machine with multi-supporting}

The body, rollers, wheels, and bearings of the large-scale rotary machine can be considered as a chain multibody system. By using TMM, the body system may be divided into a certain number of subsystems, which can be represented by various elements including rigid disks, elastic shafts, and linear springs, as shown in Fig. 2 . The head of rotary machine is the coordinate system origin, the straight axis line is $x$ axes, the vertical direction of the supporting section is $y$ axes, and the horizontal direction is $z$ axes. For any subsystem $i$, all elements are limited to a plane motion, $y$ and $z$ plane. 


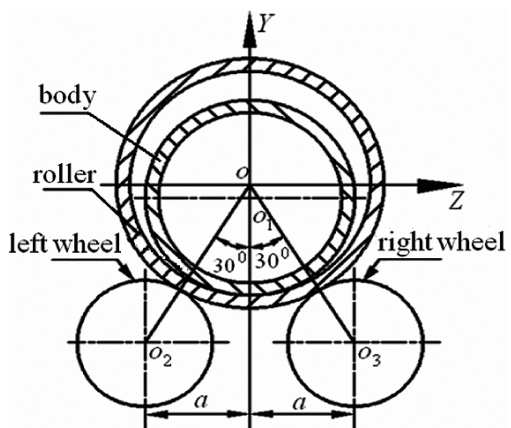

(a) Geometry model

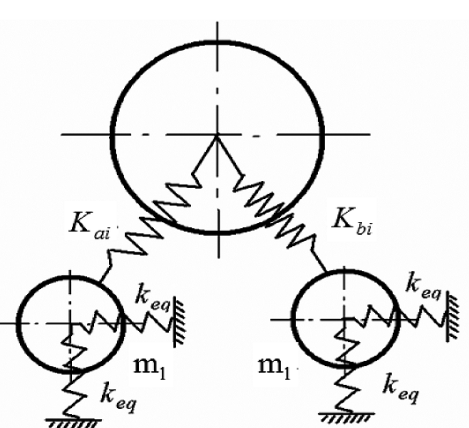

(b) Mechanic model

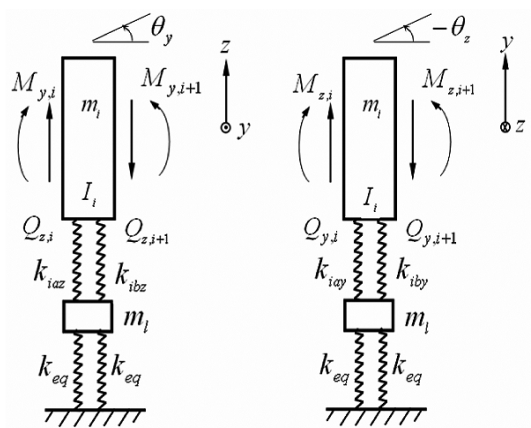

(c) The sketch in $y$ and $z$ plane

Fig. 3. The geometry and mechanic model of wheel-bearing supporting structure.

\subsection{Stiffness of multiple wheel-bearing supporting structures}

Multiple wheel-bearing supporting structures are represented by linear springs in system discrete model, as shown in Fig. 2. For rotary machine, each supporting structure is composed of a roller set around the body, two wheels located on the left and the right of roller at $30^{\circ}$ angle with vertical line, and four shafts and bearings, as shown in Fig. 3(a). The supporting structures are simplified as springs, as shown in Fig. 3(b). For any subsystem $i$ composed of a rigid body with linear springs(Shown in Fig. 3(c)), $m_{i}$ represents the weight of the rigid disk, $k_{i a y}$ and $k_{i a z}$ denote the supporting stiffness of the right wheels in $y$ and $z$ axis, $k_{i b y}$ and $k_{i b z}$ are the supporting stiffness of the left wheels in $y$ and $z$ axis, $\mathrm{k}_{\mathrm{eq}}$ is the equivalent stiffness of the journal bearing, $\mathrm{m}_{\mathrm{l}}$ is the wheel weight. The equivalent stiffness of supporting structures can be deduced by using their difference motion equation.

For the mechanic model of the supporting structure in Fig. 3(c), the equivalent stiffness of the supporting structure is anisotropy. By ignoring the coupling terms of $y$ and $z$ direction, the mass and stiffness can be written in matrix form

$$
\mathbf{M}_{l}=\left[\begin{array}{cc}
\mathrm{m}_{\mathrm{l}} & 0 \\
0 & \mathrm{~m}_{\mathrm{l}}
\end{array}\right] ; \mathbf{K}_{a i}=\left[\begin{array}{cc}
k_{a z} & 0 \\
0 & k_{a y}
\end{array}\right]_{i} ; \mathbf{K}_{b i}=\left[\begin{array}{cc}
k_{b z} & 0 \\
0 & k_{b y}
\end{array}\right]_{i} ; \mathbf{K}=\left[\begin{array}{cc}
\mathbf{k}_{\mathrm{eq}} & 0 \\
0 & \mathrm{k}_{\mathrm{eq}}
\end{array}\right]
$$

In the supporting structure, wheel, shaft and journal bearings can be considered as a rigid disk with a elastic spring, because the journal bearing with overload and low speed can not generate oil film $[18,19]$. Therefore, the stiffness $\mathrm{k}_{\mathrm{eq}}$ can be easily written as

$$
\mathrm{k}_{\mathrm{eq}}=\mathrm{k}_{\mathrm{l}} \mathrm{k}_{\mathrm{p}} /\left(\mathrm{k}_{\mathrm{l}}+\mathrm{k}_{\mathrm{p}}\right)
$$

where $\mathrm{k}_{\mathrm{l}}$ is the shaft stiffness, $\mathrm{k}_{\mathrm{p}}$ is the bearing stiffness.

Supposed the displacement vector of body is $\mathbf{D}=[y, z]^{T}$, the displacement vectors of the left and right wheel are $\mathbf{D}_{l}=\left[y_{l}, z_{l}\right]^{T}$ and $\mathbf{D}_{r}=\left[y_{r}, z_{r}\right]^{T}$, respectively. The motion of the supporting structure can be expressed as

$$
\mathbf{M}_{l} \ddot{\mathbf{D}}+\mathbf{K}_{a i} \mathbf{D}_{l}+\mathbf{K}_{b i} \mathbf{D}_{r}-\mathbf{K}_{e q}\left(\mathbf{D}-\mathbf{D}_{l}-\mathbf{D}_{r}\right)=0
$$

For natural vibration of the body system, the solution of the above equation can be assumed as

$$
\mathbf{D}=D e^{s t} \quad \mathbf{D}_{l}=D_{l} e^{s t} \quad \mathbf{D}_{r}=D_{r} e^{s t}
$$

When neglecting damper effects, $S=i \beta, \beta$ is the natural frequency of the body system, $\beta_{k}(k=1,2, \ldots)$ denotes $k^{\text {th }}$ natural frequency. Then, the equivalent stiffness matrices of supporting structure can be expressed as

$$
\begin{aligned}
& \mathbf{K}_{s a i}=\mathbf{K}_{e q}\left[\mathbf{K}_{e q}+\mathbf{K}_{a i}+S^{2} \mathbf{M}_{l}\right]^{-1}\left[\mathbf{K}_{a i}+S^{2} \mathbf{M}_{l}\right] \\
& \mathbf{K}_{s b i}=\mathbf{K}_{e q}\left[\mathbf{K}_{e q}+\mathbf{K}_{b i}+S^{2} \mathbf{M}_{l}\right]^{-1}\left[\mathbf{K}_{b i}+S^{2} \mathbf{M}_{l}\right]
\end{aligned}
$$

where $\mathbf{K}_{s a i}, \mathbf{K}_{s b i}$ denote the complex equivalent stiffness matrices of the $i^{\text {th }}$ left and right wheel-bearing structure, respectively.

Let $\mathbf{K}_{i}$ denotes the total stiffness of the two sides supporting structures, as yielded as

$$
\mathbf{K}_{i}=\mathbf{K}_{s a i}+\mathbf{K}_{s b i}
$$




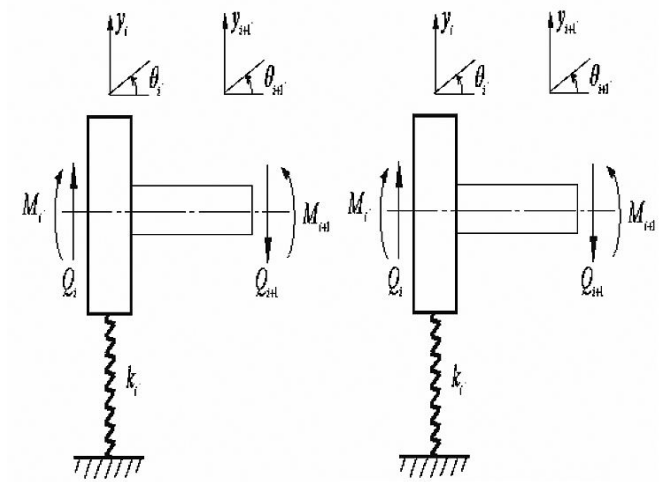

(a) The subsystem combined three elements

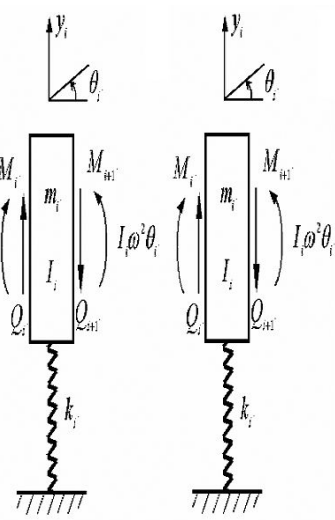

(b) A disk with a spring

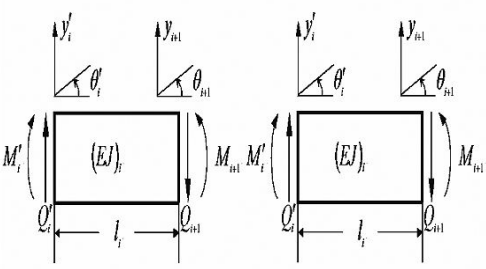

(c) Elastic shaft

Fig. 4. Schematic diagram of the subsystem and elements.

\subsection{State vectors, transfer equations and transfer matrices of elements}

The state vectors of any element end are defined as

$$
\mathbf{Z}_{i}=\left[z, \theta_{y}, M_{y}, Q_{z}, y,-\theta_{z},-M_{z}, Q_{y}\right]_{i}^{T}
$$

where $y, z$ are the point coordinates of the body rotary center, $\theta_{y}, \theta_{z}$ are corresponding angular displacements, $M_{y}, M_{Z}$ are the corresponding internal torques, $Q_{y}, Q_{Z}$ are the corresponding internal forces, subscript $i$ is the subsystem indices, and superscript $T$ denotes the matrix transpose.

The transfer equation and the transfer matrix $\mathbf{T}_{i}$ between the divided body subsystems can be written as

$$
\mathbf{Z}_{i}=\mathbf{T}_{i} \mathbf{Z}_{i-1}
$$

The transfer equation describes the mutual relationship between the state vectors at two ends of the $i$ th subsystem. For rotary machine, any subsystem is composed of three elements, namely, a rigid disk with a linear spring, and an elastic shaft. Figure 4 shows a subsystem combined three elements, a rigid disk with a elastic spring, and an elastic shaft respectively.

According to D'Alembert's principle, the dynamic equation of a disk with a spring (Seen in Fig. 4(b)) can be written as

$$
\left\{\begin{array}{l}
Q_{y, i+1}=Q_{y, i}-m_{i} S^{2} y_{i}-k_{y y, i} y_{i} \\
Q_{z, i+1}=Q_{z, i}-m_{i} S^{2} z_{i}-k_{z z, i} z_{i} \\
M_{y, i+1}=M_{y, i}-I_{i} S^{2} \theta_{y, i} \\
M_{z, i+1}=M_{z, i}-I_{i} S^{2} \theta_{z, i}
\end{array}\right.
$$

where $k_{y y}, k_{z z}$ are the total stiffness of the supporting structure in $y$ and $z$ axis, namely diagonal terms of $\mathbf{K}_{i}, I_{i}$ is the moment of inertia.

Therefore, transfer matrix for this element can be written as

$$
\mathbf{Z}_{i+1}=\mathbf{D}_{i} \mathbf{Z}_{i}
$$

$$
\mathbf{D}_{i}=\left(\begin{array}{cccccccc}
1 & 0 & 0 & 0 & 0 & 0 & 0 & 0 \\
0 & 1 & 0 & 0 & 0 & 0 & 0 & 0 \\
0 & -I_{i} S^{2} & 1 & 0 & 0 & 0 & 0 & 0 \\
-m_{i} S^{2}-k_{z z} & 0 & 0 & 1 & 0 & 0 & 0 & 0 \\
0 & 0 & 0 & 0 & 1 & 0 & 0 & 0 \\
0 & 0 & 0 & 0 & 0 & 1 & 0 & 0 \\
0 & 0 & 0 & 0 & 0 & -I_{i} S^{2} & 1 & 0 \\
0 & 0 & 0 & 0 & -m_{i} S^{2}-k_{y y} & 0 & 0 & 1
\end{array}\right)
$$


As rotary machine generally operates at low speed, the gyroscopic effects are not included in the above equation.

For the elastic shaft without mass (Seen in Fig. 4(c)), transfer matrix $\mathbf{B}_{i}$ is written as

$$
\mathbf{B}_{i}=\left(\begin{array}{cccccccc}
1-l & l & l^{2} / 2 E J & l^{3} / 6 E J & 0 & 0 & 0 & 0 \\
0 & 1 & l / E J & l^{2} / 2 E J & 0 & 0 & 0 & 0 \\
0 & 0 & 1 & l & 0 & 0 & 0 & 0 \\
0 & 0 & 0 & 1 & 0 & 0 & 0 & 0 \\
0 & 0 & 0 & 0 & 1 & l & l^{2} / 2 E J & l^{3} / 6 E J \\
0 & 0 & 0 & 0 & 0 & 1 & l / E J & l^{2} / 2 E J \\
0 & 0 & 0 & 0 & 0 & 0 & 1 & l \\
0 & 0 & 0 & 0 & 0 & 0 & 0 & 1
\end{array}\right)
$$

where $l$ is the corresponding length of elastic shaft, $E$ is the elastic modulus, $J$ is second moment of area.

Therefore, for any subsystem $i$ composed of a disk with a spring, and a elastic shaft (Seen in Fig. 4(a)), transfer matrix can be obtained according to the mechanics relationship of material

$$
\begin{aligned}
\mathbf{T}_{i}= & \mathbf{B}_{i} \mathbf{D}_{i}=\left(\begin{array}{ll}
\mathbf{t}_{11} & \mathbf{t}_{12} \\
\mathbf{t}_{21} & \mathbf{t}_{22}
\end{array}\right)_{i} \\
\mathbf{t}_{12 i}= & \mathbf{t}_{21 i}=0 \\
\mathbf{t}_{11 i}= & \left(\begin{array}{cccc}
1-\frac{l^{3}}{6 E J}\left(m S^{2}+K_{z z}\right) & l+\frac{l^{2}}{2 E J} I S^{2} & \frac{l^{2}}{2 E J} & \frac{l^{3}}{6 E J} \\
-\frac{l^{2}}{2 E J}\left(m S^{2}+K_{z z}\right) & 1+\frac{l}{E J} I S^{2} & \frac{l}{E J} & \frac{l^{2}}{2 E J} \\
-l\left(m S^{2}+K_{z z}\right) & I S^{2} & 1 & l \\
-\left(m S^{2}+K_{z z}\right) & 0 & 0 & 1
\end{array}\right)_{i} \\
\mathbf{t}_{22 i}= & \left(\begin{array}{cccc}
1-\frac{l^{3}}{6 E J}\left(m S^{2}+K_{y y}\right) & l+\frac{l^{2}}{2 E J} I S^{2} & \frac{l^{2}}{2 E J} & \frac{l^{3}}{6 E J} \\
-\frac{l^{2}}{2 E J}\left(m S^{2}+K_{y y}\right) & 1+\frac{l}{E J} I S^{2} & \frac{l}{E J} & \frac{l^{2}}{2 E J} \\
-l\left(m S^{2}+K_{y y}\right) & I S^{2} & 1 & l \\
-\left(m S^{2}+K_{y y}\right) & 0 & 0 & 1
\end{array}\right)_{i}
\end{aligned}
$$

\section{Riccati transfer equation of the large-scale rotary machine with multi-supporting}

The overall transfer equation of the body system can be obtained by multiplying the transfer matrices of the subsystems

$$
\begin{aligned}
& \mathbf{Z}_{N}=\mathbf{T}_{\text {all }} \mathbf{Z}_{0} \\
& \mathbf{T}_{\text {all }}=\mathbf{T}_{N} \mathbf{T}_{N-1} \ldots \mathbf{T}_{i} \ldots \mathbf{T}_{2} \mathbf{T}_{1}
\end{aligned}
$$

where $\mathbf{T}_{\text {all }}$ is the overall transfer matrix of the body system, $\mathbf{Z}_{0}, \mathbf{Z}_{N}$ are the state vectors of boundaries of the body, subscript $i$ denotes the subsystem indices.

Substituting boundaries state vectors into Eq. (14), the natural frequency equation can be obtained. Then solving the natural frequency equation, the natural frequencies can be gained. Using the transfer equations of subsystem Eq. (8) for each natural frequencies, the state vector of any end of subsystem can be obtained easily. However, with the increase of the number $N$ of the subsystems, rounding errors are magnified by multiplying transfer matrices, even make the computation results invalid. The Riccati transfer matrix method is an important way to improve the numerical stability $[16,19,22]$.

When using Riccati transfer matrix method, the state vectors are divided into two parts

$$
\mathbf{Z}_{i}=[\mathbf{f}, \mathbf{e}]_{i}^{T}=\left[M_{y}, Q_{z},-M_{z}, Q_{y}, y,-\theta_{z}, z, \theta_{y}\right]_{i}^{T}
$$

where $\mathbf{f}_{i}$ includes the state variables that are zero on the boundary of the body systems, $\mathbf{e}_{i}$ includes the other unknown variables. 
Therefore, Eq. (8) can be yielded as

$$
\begin{aligned}
& {\left[\begin{array}{l}
\mathbf{f} \\
\mathbf{e}
\end{array}\right]_{i+1}=\left[\begin{array}{ll}
\mathbf{u}_{11} & \mathbf{u}_{12} \\
\mathbf{u}_{21} & \mathbf{u}_{22}
\end{array}\right]_{i}\left[\begin{array}{l}
\mathbf{f} \\
\mathbf{e}
\end{array}\right]_{i}} \\
& \mathbf{u}_{11 i}=\left(\begin{array}{cccc}
1 & l & 0 & 0 \\
0 & 1 & 0 & 0 \\
0 & 0 & 1 & l \\
0 & 0 & 0 & 1
\end{array}\right)_{i} \quad \mathbf{u}_{12 i}=\left(\begin{array}{cccc}
-l\left(m S^{2}+K_{z z}\right) & I S^{2} & 0 & 0 \\
-\left(m S^{2}+K_{z z}\right) & 0 & 0 & 0 \\
0 & 0 & -l\left(m S^{2}+K_{y y}\right) & I S^{2} \\
0 & 0 & -\left(m S^{2}+K_{y y}\right) & 0
\end{array}\right)_{i} \\
& \mathbf{u}_{21 i}=\left(\begin{array}{cccc}
\frac{l^{2}}{2 E J} & \frac{l^{3}}{6 E J} & 0 & 0 \\
\frac{l}{E J} & \frac{l^{2}}{2 E J} & 0 & 0 \\
0 & 0 & \frac{l^{2}}{2 E J} & \frac{l^{3}}{6 E J} \\
0 & 0 & \frac{l}{E J} & \frac{l^{2}}{2 E J}
\end{array}\right) \\
& \mathbf{u}_{22 i}=\left(\begin{array}{cccc}
1-\frac{l^{3}}{6 E J}\left(m S^{2}+K_{y y}\right) & l+\frac{l^{2}}{2 E J} I S^{2} & 0 & 0 \\
-\frac{l^{2}}{2 E J}\left(m S^{2}+K_{y y}\right) & 1+\frac{l}{E J} I S^{2} & 0 & 0 \\
0 & 0^{2} & 1-\frac{l^{3}}{6 E J}\left(m S^{2}+K_{z z}\right) & l+\frac{l^{2}}{2 E J} I S^{2} \\
0 & 0 & -\frac{l^{2}}{2 E J}\left(m S^{2}+K_{z z}\right) & 1+\frac{l}{E J} I S^{2}
\end{array}\right)_{i}
\end{aligned}
$$

Introducing the Riccati transform

$$
\mathbf{f}_{i}=\mathbf{R}_{i} \mathbf{e}_{i}
$$

Substituting it into Eq. (16), we obtain

$$
\begin{aligned}
& \mathbf{e}_{i}=\left[\mathbf{u}_{21} \mathbf{R}+\mathbf{u}_{22}\right]_{i}^{-1} \mathbf{e}_{i+1} \\
& \mathbf{f}_{i+1}=\left[\mathbf{u}_{11} \mathbf{R}+\mathbf{u}_{12}\right]_{i}\left[\mathbf{u}_{21} \mathbf{R}+\mathbf{u}_{22}\right]_{i}^{-1} \mathbf{e}_{i+1}
\end{aligned}
$$

By comparing Eqs (19) with (17), the transfer formula can be found as

$$
\mathbf{R}_{i+1}=\left[\mathbf{u}_{11} \mathbf{R}+\mathbf{u}_{12}\right]_{i}\left[\mathbf{u}_{21} \mathbf{R}+\mathbf{u}_{22}\right]_{i}^{-1}
$$

According to the boundary conditions, matrices $\mathbf{f}, \mathbf{e}$, and Ron boundaries can be determined as

$$
\mathbf{f}_{0}=0, \quad \mathbf{e}_{0} \neq 0, \quad \mathbf{R}_{0}=0, \quad \mathbf{f}_{N}=0, \quad \mathbf{e}_{N} \neq 0
$$

By using Eq. (20) repeatedly, the matrix $\mathbf{R}_{i}$ at any subsystem $i$ can be obtained. At the last subsystem $N$ of the boundary can be substituted into Eq. (17), and we can get

$$
\mathbf{f}_{N}=\mathbf{R}_{N} \mathbf{e}_{N}
$$

According to the boundary conditions, we get the natural frequency equation

$$
|\mathbf{R}|_{N}=\left|\begin{array}{ll}
\mathbf{R}_{11} & \mathbf{R}_{12} \\
\mathbf{R}_{21} & \mathbf{R}_{22}
\end{array}\right|_{N}=0
$$

By solving Eq. (23), the natural frequencies can be obtained. The dichotomy method is adopted to search the natural frequencies $\beta_{k}(k=1,2 \ldots n)$ which is approximately satisfied Eq. (23). For each natural frequency $\beta_{k}$, using the overall transfer equation of the body system and the transfer equations of elements, the state vector of any point as well as modal shape of the body system can be obtained easily. 


\section{Response vibration analysis of the large-scale rotary machine with multi-supporting}

In practice, the body vibration of rotary machine is excited by interior factors causing axis line deflection and exterior forces. The interior factors, common asymmetric geometry of major parts caused by manufacture, abrasion, and expansion difference, will result in dynamical change of rotary centre, thus the corresponding element state vector is changed $[8,10,28]$. The exterior forces are introduced from driveline vibration, material variety, abnormal operation, and machine start/stop, etc. [29,30]. The above excitation will introduce either displacement vectors $(\Delta y, \Delta z)$ or force vectors $\left(\Delta Q_{y}, \Delta Q_{z}\right)$ to corresponding element state. The body response can also be analyzed by using Riccati-TMM. The state vector of the other end of the excited element is rewritten as

$$
\left[\begin{array}{l}
\mathbf{f} \\
\mathbf{e}
\end{array}\right]_{i+1}=\left[\begin{array}{ll}
\mathbf{u}_{11} & \mathbf{u}_{12} \\
\mathbf{u}_{21} & \mathbf{u}_{22}
\end{array}\right]_{i}\left[\begin{array}{l}
\mathbf{f} \\
\mathbf{e}
\end{array}\right]_{i}+\left[\begin{array}{l}
\overline{\mathbf{f}} \\
\overline{\mathbf{e}}
\end{array}\right]_{i}
$$

where $\overline{\mathbf{f}}_{i}$ and $\overline{\mathbf{e}}_{i}$ is the exciting corresponding vectors.

The Riccati transform is yielded as

$$
\mathbf{f}_{i}=\mathbf{R}_{i} \mathbf{e}_{i}+\mathbf{p}_{i}
$$

Substituting it into Eq. (24)

$$
\begin{aligned}
& \mathbf{R}_{i+1}=\left[\mathbf{u}_{11} \mathbf{R}+\mathbf{u}_{12}\right]_{i}\left[\mathbf{u}_{21} \mathbf{R}+\mathbf{u}_{22}\right]_{i}^{-1} \\
& \mathbf{p}_{i+1}=\left[\mathbf{u}_{11} \mathbf{p}+\overline{\mathbf{f}}\right]_{i}-\mathbf{R}_{i+1}\left[\mathbf{u}_{21} \mathbf{p}+\overline{\mathbf{e}}\right]_{i} \\
& \mathbf{e}_{i}=\left[\mathbf{u}_{21} \mathbf{R}+\mathbf{u}_{22}\right]_{i}^{-1} \mathbf{e}_{i+1}-\left[\mathbf{u}_{21} \mathbf{R}+\mathbf{u}_{22}\right]_{i}^{-1}\left[\mathbf{u}_{21} \mathbf{p}+\overline{\mathbf{f}}\right]_{i}
\end{aligned}
$$

By using Eqs (26) and (27) repeatedly, the matrix $\mathbf{p}_{i}$ at any subsystem $i$ can be obtained. Considering the last element end $N$ of the boundary, $\mathbf{R}_{N}$ and $\mathbf{p}_{N}$ are substituted into Eq. (25), then we can get the overall motion equation

$$
\mathbf{f}_{N}=\mathbf{R}_{N} \mathbf{e}_{N}+\mathbf{p}_{N}=0
$$

Equation (29) indicates the relationship between the state variables at the boundaries of the body system. In general, the unknown $\mathbf{e}_{N}$ can be obtained by solving the above equation by replacing $\beta$ with the rotary speed of the body. According to the transfer matrix Eq. (16), the response values of state vectors can be obtained.

\section{Practical application}

\subsection{Dynamics model of rotary kiln}

We computed the natural frequencies of the body system of rotary kiln, as well as the response with axis line deflection of the supporting structure. The rotary kiln is shown in Fig. 5(a). The body length and radius are 100m and $4 \mathrm{~m}$ respectively, the total weight is $950 \times 10^{4} \mathrm{~N}$. The kiln has 5 supporting structures, located $6.3 \mathrm{~m}, 24.3 \mathrm{~m}$, $44.1 \mathrm{~m}, 67.5 \mathrm{~m}$, and $89.1 \mathrm{~m}$, from kiln head, respectively. The kiln is divided into various elements according to body structure, as shown in Fig. 5(b). Any subsystem is composed of rigid disks, elastic shafts, and linear springs. The dynamics model of the body system is a chain multibody system composed of 12 subsystems, namely, 12 rigid body elements, 12 elastic shaft elements, and 5 linear spring elements. Supposed $5 \mathrm{~mm}$ axis line deflection on the third supporting structure Y direction is applied for response analysis of the body system, as shown in Fig. 5(b).

According to the material and geometry properties of the rotary kiln, the values for the computational parameters of transfer matrix equations are as follows.

$l_{1}=5.3 \mathrm{~m}, l_{3}=16 \mathrm{~m}, l_{5}=17.8 \mathrm{~m}, l_{7}=3.5 \mathrm{~m}, l_{8}=17.9 \mathrm{~m}, l_{10}=19.6 \mathrm{~m}, l_{12}=9.9 \mathrm{~m}, l_{2}=l_{4}=l_{6}=l_{9}=l_{11}=$ $2 \mathrm{~m} ; m_{1}=580.5 \mathrm{KN}, m_{2}=343 \mathrm{KN}, m_{3}=1902.5 \mathrm{KN}, m_{4}=440 \mathrm{KN}, m_{5}=1661.5 \mathrm{KN}, m_{6}=m_{9}=$ $370 \mathrm{KN}, m_{7}=600 \mathrm{KN}, m_{8}=1432 \mathrm{KN}, m_{10}=1581.5 \mathrm{KN}, m_{11}=260 \mathrm{KN}, m_{12}=247.5 \mathrm{KN} ; I_{i}=4 * m_{i}$; $E J_{1}=E J_{3}=E J_{5}=E J_{8}=E J_{10}=E J_{12}=125 \mathrm{GN} . \mathrm{m} . \mathrm{m}, E J_{2}=E J_{4}=E J_{6}=E J_{7}=E J_{9}=E J_{11}=$ 250 GN. m .m;

Anisotropy stiffness of the supporting structures in $y$ and $z$ direction are given as follows.

$y$ direction: $k_{y y 1}=6.5 \times 10^{4} \mathrm{~N} / \mathrm{mm}, k_{y y 3}=36 \times 10^{4} \mathrm{~N} / \mathrm{mm}, k_{y y 5}=39.08 \times 10^{4} \mathrm{~N} / \mathrm{mm}, k_{y y 8}=$ $22.46 \times 10^{4} \mathrm{~N} / \mathrm{mm}, k_{y y 10}=3.78 \times 10^{4} \mathrm{~N} / \mathrm{mm}$

$z$ direction: $k_{z z 1}=3.76 \times 10^{4} \mathrm{~N} / \mathrm{mm}, k_{z z 3}=20.78 \times 10^{4} \mathrm{~N} / \mathrm{mm}, k_{z z 5}=20.56 \times 10^{4} \mathrm{~N} / \mathrm{mm}, k_{z z 8}=$ $12.96 \times 10^{4} \mathrm{~N} / \mathrm{mm}, k_{z z 10}=2.18 \times 10^{4} \mathrm{~N} / \mathrm{mm}$ 
Table 1

First five order natural frequencies $\beta_{k}$ of kiln (r/min)

\begin{tabular}{cccccc}
\hline Modal & $\beta_{1}$ & $\beta_{2}$ & $\beta_{3}$ & $\beta_{4}$ & $\beta_{5}$ \\
\hline TMM & 28.5 & 51.5 & 60.7 & 145.4 & 148.5 \\
Riccati TMM & 28.5 & 51.4 & 59.8 & 145.2 & 147.5 \\
\hline
\end{tabular}

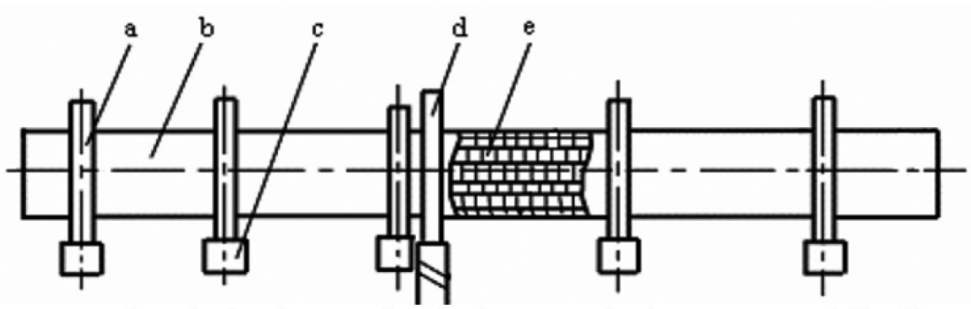

a. roller b. body c. wheel d. transmission gear e. kiln line

(a) The sketch of rotary kiln

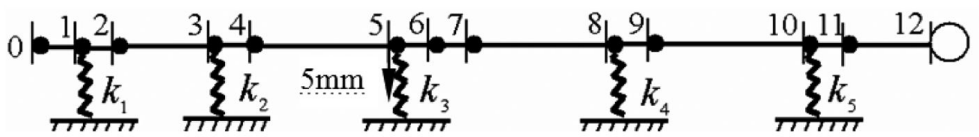

(b) Dynamics model of the body system

Fig. 5. The sketch of rotary kiln and dynamics model of the body system.

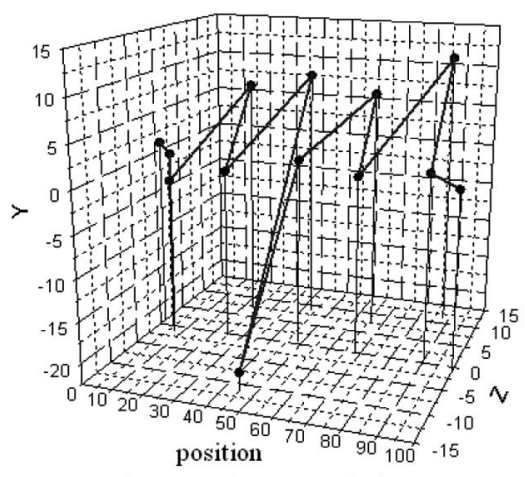

a. First order modal curve

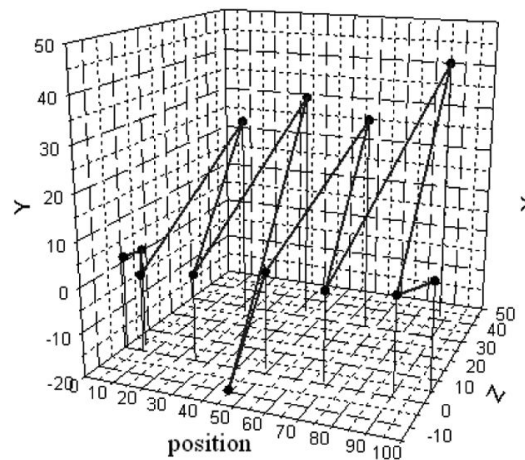

b. Second order modal curve

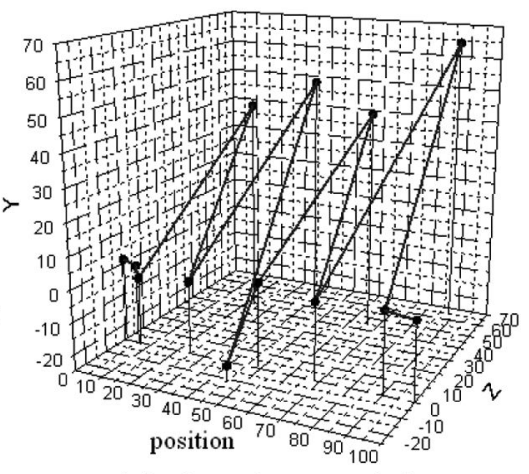

c. Third order modal curve

Fig. 6. First three modal curve of the body system.

\subsection{Natural frequencies of rotary kiln}

The results of the natural frequencies $\beta_{k}(k=1,2,3, \ldots)$ of the body system are shown in Table 1 . The first three modal curves are shown in Fig. 6. It can be seen clearly from Table 1 that the results of natural frequencies get by Riccati-TMM and by TMM have good agreements, which mutually validating the dynamics model, numerical accuracy in this paper.

As rotary speed of rotary kiln is about $3 \mathrm{r} / \mathrm{min}$, it is known form Table 1 that the kiln do not need to pass resonance frequency, so there is no resonance when start/stop the machine. The first three orders modal curves are shown in Fig. 6. The practical track is ellipse with long axis $y_{i}$ and short axis $z_{i}$.

\subsection{Dynamic response of kiln body system}

The dynamics response of the body system with $5 \mathrm{~mm}$ axis line deflection on the third supporting structure $\mathrm{Y}$ direction is simulated with Riccati TMM. In order to know the axis line vibration condition, it is important to know 


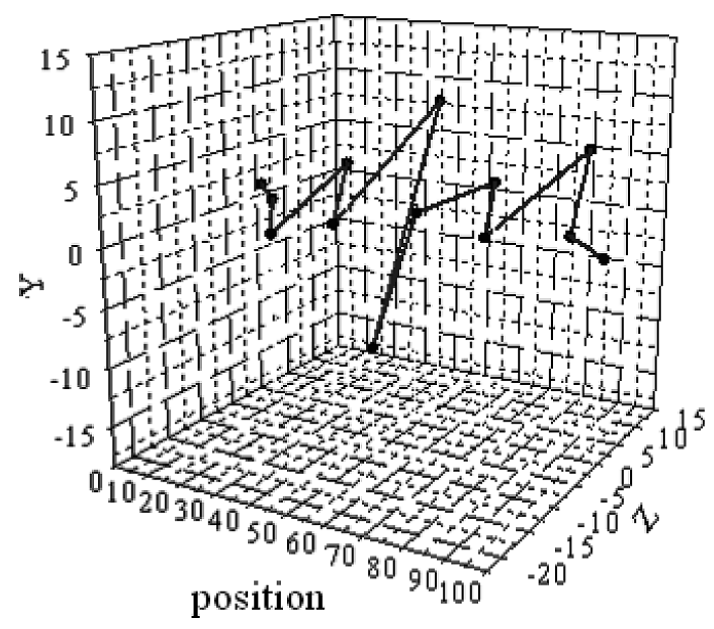

Fig. 7. Displacement response of the body system.

the displacement response, as shown in Fig. 7.

Some conclusions are drawn as follows:

(1) The first three orders modal curves are polygonal lines. With the increase of the natural frequencies, each polygonal line become steeper, and body vibration become stronger.

(2) Vibration values in supporting structure position are opposite to the others positions, and vibration nodes are not in supporting positions. Therefore, dynamical errors of the common axis line measurement system are produced as their measurements generally conduct in the supporting positions. Modal analysis results provide important theory to develop accuracy axis line measurement system with dynamical error compensation.

(3) Vibration amplitudes in the middle of elastic bodies limited between two supporting structures are too large to ignore. Fatigue damage should include these dynamic stresses in structure strength analysis and design. Furthermore, body vibration will bring exciting forces to other parts, which may accelerate uneven fatigue damage.

\section{Conclusions}

The dynamics of the large-scale rotary machine with multiple wheel-bearing structures is investigated in this paper. Based on the Riccati transfer matrix method, the transfer matrices and overall transfer equation are developed to calculate natural frequencies, and response overall motion equation is established for response analysis. Multiple wheel-bearing structures are simplified as linear springs, and their anisotropy equivalent stiffness are deduced. Taken a rotary kiln as an instance, natural frequencies, modal curves, and response vibration are obtained by solving Eqs (23) and (29). The body vibration modal curves illustrate the cause of dynamical errors in common axis line measurement methods. The displacement response can be used for further measurement dynamical error analysis and compensation. The response overall motion equation could be applied to predict the body motion under abnormal mechanics condition, and provide theory guidance for machine failure diagnosis.

\section{Acknowledgements}

The authors would like to acknowledge the support of the National High-tech R\&D Program "863", Grant NO.2007AA04Z415, the National Natural Science Foundation of China, Grant NO.50675066, and Scientific Research Fund of Hunan Provincial Education Department, Grant NO.09C407. 


\section{References}

[1] X.J. Li and Y.L. Liu, Rotary health maintenance theory and technology, China Machine Press, 2005.

[2] X.J. Li, L.L. Jiang et al., Research on supporting load distribution of large-scale rotary kiln with multi-support and variable-stiffness, Chinese Journal of Computational Mechanics 2(22) (2005), 207-213.

[3] Y. Zhang and Z.X. Li, Dynamic measuring and adjustment on-line on rotary kiln, China Building Material Equipment 3 (2002), 3-7.

[4] Y.G. Xiao and Y.L. Liu, Axial deviation and roller adjustment on rotary kiln, Cement Technology (2) (2002), 30-32.

[5] Z.Y. Zhou, Report about axes of $\Phi 4.7 \times 75 \mathrm{M}$ rotary kiln in different temperature, China Building Material Equipment (1) (1998), $21-24$.

[6] E. Bingham and F. Morrison, Plants decrease kiln operating costs using proven preventative maintenance techniques, World Cement 24(12) (1993), 40-41.

[7] Y. Zhang, Increasing plant availability by mechanical checking of the cement rotary kiln axis, Journal of Wuhan University of Technology 16(3) (2001), 76-78.

[8] N.K. Andersen, Mechanical monitoring and maintenance of rotary kilns and coolers, Zement-Kalk-Gips International 39(8) (1986), $447-456$.

[9] W.H. Gebhart, Kiln alignment analysis: the direct method, Global Cement and Lime Magazine (11) (2001), $21-24$.

[10] W.H. Gebhart and V.L. Roxanne, Kiln alignment and shell ovality, a new approach, World Cement 22(7) (1991), 4-9.

[11] W. Schiehlen, Multibody system dynamics: roots and perspectives, Multibody Syst Dyn 1 (1997), 149-188.

[12] W. Schiehlen, Research trends in multibody system dynamics, Multibody Syst Dyn 18 (2007), 3-13.

[13] E.C. Pestel and F.A. Leckie, Matrix Method in Elastomechanics, McGraw-Hil1, New York, 1963.

[14] H. Holzer, Die Berechnung der drehsenwingungen, Springer, Berlin, 1921.

[15] M.A. Prohl, A general method for calculating critical speeds of a flexible rotor in fluid film bearings, J of Appl Mech Trans ASME 12(3) (1945), 142-148.

[16] G.C. Horner and W.D. Pilkey, The Riccati transfer matrix method, J Mech Des 1 (1978), 297-302.

[17] M.A. Dokanish, A new approach for plate vibration: combination of transfer matrix and finite element technique, $J$ Mech Des 94 (1972), 526-530.

[18] A.S. Kumar and T.S. Sankar, A new transfer matrix method for response analysis of large dynamic systems, Comput Struct 23 (1986), 545-552.

[19] H.Y. Xue, A combined dynamic finite element Riccati transfer matrix method for solving non-linear eigenproblems of vibrations, Computers \& Structures 53 (1994), 1257-1261.

[20] R.G Loewy and N. Bhntani, Combined finite element-transfer matrix method, Journal of Sound and Vibration 226(5) (1999), 1048-1052.

[21] X.T. Rui, B. He, B. Rong et al., Discrete time transfer matrix method for multi-rigid-flexible-body system moving in plane, Journal of Multi-Body Dynamics 223(K1) (2009), 23-42.

[22] B. He, X.T. Rui and G.P. Wang, Riccati discrete time transfer matrix method for elastic beam undergoing large overall motion, Multibody Syst Dyn 18 (2007), 579-598.

[23] X.T. Rui, G.P. Wang et al., Transfer matrix method for linear multibody system, Multibody Syst Dyn 19 (2008), $179-207$.

[24] B. Rong, X.T. Rui and G.P. Wang, New method for dynamics modeling and analysis on flexible plate undergoing large overall motion, Multi-body Dyn 224(K1) (2010), 33-44.

[25] B. Rong, X.T. Rui, F.F. Yang et al., Discrete time transfer matrix method for dynamics of multibody system with real-time control, Journal of Sound and Vibration 329(6) (2010), 627-643.

[26] S. Salazar, H. Romero et al., Modeling and real-time stabilization of an aircraft having eight rotors, J Intell Robot Syst 54 (2009), $455-470$.

[27] C.Y. Tsai and S.C. Huang, Transfer matrix for rotor coupler with parallel misalignment, Journal of Mechanical Science and Technology 23 (2009), 1383-1395.

[28] T. Suezawa, T. Shimizu and E. Nakajima, Ovality of cement rotary kiln in operation, Journal of the Technical Association of Refractories 23(1) (2003), 57-61.

[29] S. Uchida and H. Sugiyama, Numerical analysis of stress and displacement of rotary kiln bricks under thermal and mechanical loads, Taikabutsu Overseas 16 (4) (2003), 114.

[30] S.K. Li, Rotary kiln transmission gear vibration analysis and treatment method, Light Metal 9 (2003), 54-56. 

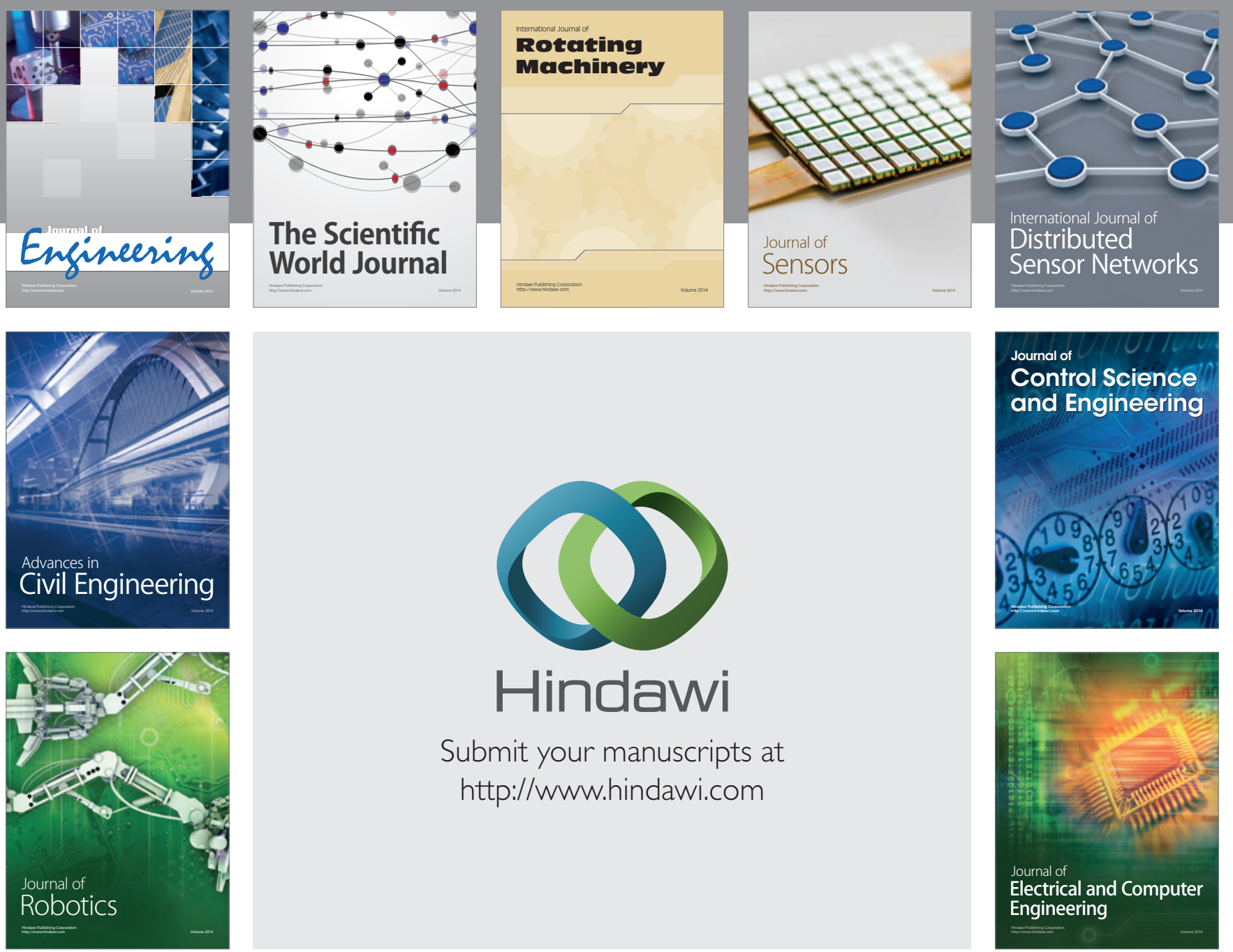

Submit your manuscripts at

http://www.hindawi.com
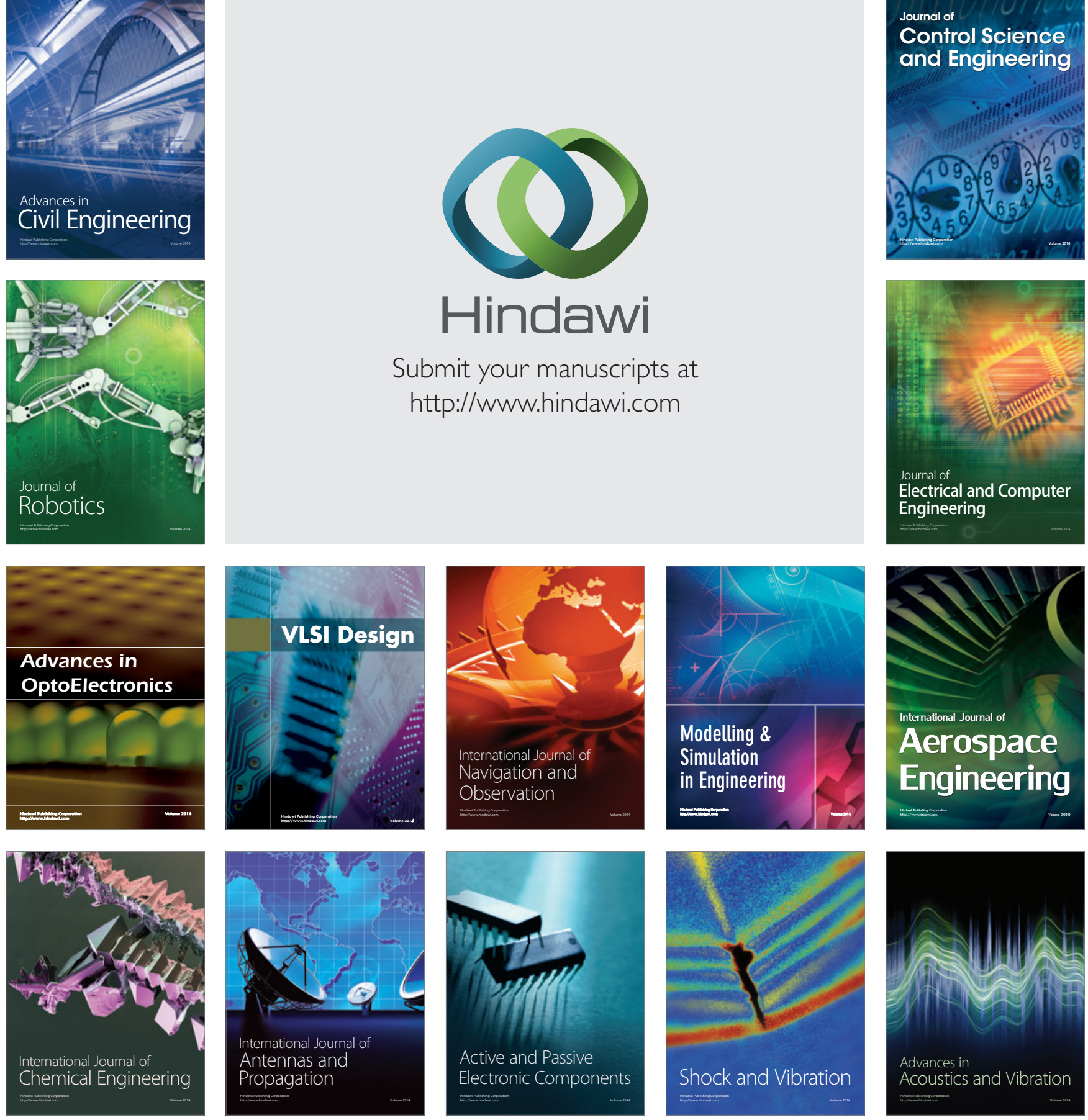